\title{
Numerical simulation of pressure relief gas flow under mining conditions
}

\author{
Wentao Liao ${ }^{1}$, Xiaoyu Deng ${ }^{2 *}$ \\ ${ }^{1}$ China University of Mining and Technology, Beijing 100083, China \\ ${ }^{2}$ Guizhou University of Finance and Economics, Guizhou 550001, China \\ Email: dengxiaoyu550@163.com
}

\begin{abstract}
This paper aims to disclose the features of pressure relief gas (PRG) flow in coal-rock mass surrounding the coal face. To this end, the basic equations of the PRG flow were established based on fluid mechanics. Relying on these equations, the PRG flow in the overlying and underlying coal-rock masses of Longfeng Coalmine was simulated on Fluent software. The simulation results show that the gas concentration in both the overlying and underlying coal-rock masses tends to increase from the intake airway to the return airway; Under the joint action of air leakage, gravitational acceleration and buoyancy, the PRG flows from the overlying and underlying coalrock masses, and accumulates near the top coal seam 1\#, the coal seam 5\# at the side of the return airway, and the bottom coal seam 13\#. The research findings shed new light on gas drainage during mining.
\end{abstract}

Keywords: Pressure Relief Gas (PRG), Buried Pipe Extraction, Numerical Simulation, Overlying and Underlying Coal-rock Masses.

\section{INTRODUCTION}

Most coal mining operations are conducted underground, and are thus subject to gas disaster, flood, and fire. Among them, gas disaster poses the greatest threat to mining safety [1].

The coal seams with a history of outbursts of coal and gas are featured by a strong gas adsorption capacity and slow rate of gas desorption [2-5]. In such coal seams, the slow gas migration makes it difficult to diversify the use of gas through the conventional drilling and drainage method. After all, the conventional approach is troubled by small gas flow, low gas concentration, and poor pumping effect.

The previous studies have shown that the occurrence of mining disasters is inseparable from the deformation of the overlying coal-rock [6-8]. Mining may result in surface subsidence and collapse, leading to eco-environment damages and personal and property losses [9]. In addition, the fractures induced by mining will increase the risk of a series of problems, such as outbursts and water inrush [10-12].

Much research has been done on the gas migration in coal seam. Taking the coal in porous medium as a uniformly distributed virtual continuum, the linear gas seepage theory holds that the gas flow in the coal seam conforms to Darcy's law on linear infiltration [13]. The gas penetration-diffusion theory views the gas flow in the coal seam as a combination of percolation and diffusion [14]. References [15-17] point out that the gas flow in coal-rock mass is heavily influenced by such factors as geo-stress, geothermal energy and electric field. In general, the studies on pressure relief gas (PRG) flow in coal seam mainly focus on the effect of the overlying coal-rock on coalface, failing to tackle the impact of gas flow in underlying coal-rock mass. The so-called PRG refers to the gas drained from the upper corner. To make up for the gap, this paper constructs a model that reveals how the coalface is influenced by the PRG flow in both the overlying and underlying coalrock masses, and discloses the features of the PRG flow around the coalface.

To control the gas drainage and utilization in mines, the establish model was applied to study the dynamic evolution of gas movement in the overlying and underlying coal-rock masses. The research direction was selected because lots of gas will flow into the goaf and accumulate in the upper corner when the mining layers are close to each other in the coal seam. In this case, it is difficult to carry out drainage during the tunneling operation, not to mention coal production, or strike a balance among "pumping, digging and mining".

The remainder of this paper is organized as follows: Section 2 introduces the mathematical model of the PRG flow around the coalface; Section 3 numerically analyses the modeling results by Fluent software; Section 4 wraps up this research with some meaningful conclusions.

\section{MATHEMATICAL MODEL OF THE PRG FLOW}

Coal is a porous medium with complex physical-mechanical features. In the coal body, the gas exists in either the adsorbed form or the free form. The conversion between the two forms is reversible by adjusting the external conditions. The gas flow is essentially the diffusion from the pore system in the coal 
body, the storage place of the adsorbed gas. With the implementation of mining, the original fractures are expanded, forming fluid channels of the free gas. Then, the gas flow is, in nature, seepage.

\subsection{PRG flow continuity equation}

According to the law of the conservation of mass, the diffusion and seepage of gas are independent from each other.

(1) Diffusion flow continuity equation

The continuity equation of the diffusion flow can be expressed as:

$$
\frac{\partial\left(\rho_{0} \phi_{0}\right)}{\partial t}=-q-\nabla \bullet\left(\rho_{0} V_{0}\right)
$$

where $\rho_{0}$ is the gas density; $\phi_{0}$ is the porosity of the coal-rock mass; $q$ is the mass of outgoing gas. The amount of mass change in the system equals the difference between the mass of incoming gas and the mass of outgoing gas.

(2) Seepage flow continuity equation

The continuity equation of the seepage flow can be expressed as:

$$
\frac{\partial(\rho \phi)}{\partial t}=q-\nabla \bullet(\rho V)
$$

After a time $\Delta \mathrm{t}$, the gas density and the porosity of the coalrock mass change under the influence of mining, resulting in a change of gas mass in the micro-body. The amount of mass change in a micro-body equals the difference between the mass of inflow minus the mass of outflow.

(3) Coupled continuity equation

The continuity equation of the diffusion flow was superposed with that of the seepage flow:

$$
\frac{\partial\left(\rho_{0} \phi_{0}\right)}{\partial t}+\nabla \bullet(\rho V)+\frac{\partial(\rho \phi)}{\partial t}+\nabla \bullet\left(\rho_{0} V_{0}\right)=0
$$

The equation depicts the coupling effect between the diffusion and seepage of the PRG. The two gas movements, both depending on the gas concentration, interact and restrict each other during the mining process.

Because of $\nabla \bullet\left(\rho_{v}\right)=\rho d v V+V g r a d \rho$ and the small size of the micro-body, the temporal variation of gas density is much greater than the spatial variation, that is, $\operatorname{Vgrad} \rho<<\phi \frac{\partial \rho}{\partial t}$.

Thus, Equation (3) can be approximated as:

$$
\rho d i v V+\phi \frac{\partial \rho}{\partial t}+\rho_{0} \operatorname{div} V_{0}+\phi_{0} \frac{\partial \rho_{0}}{\partial t}=0
$$

\subsection{Motion equation of the PRG flow}

In the coal seam, the PRG flow mainly takes place in the form of diffusion and seepage. The movement of gas in small and micro pores belongs to diffusion, while that of gas in large pores and fissures belongs to seepage. Since Darcy's law cannot describe the exact state of fluid flow at a large Reynold number $(\operatorname{Re} \geq 10)$, it is necessary to resort to the law of nonlinear seepage.

Under the action of mining, fractured zone and caving zone will appear in the coalface. Then, laminar flow, turbulent flow and transitional flow will simultaneously appear in the seepage movement of the PRG. Here, the nonlinear seepage law was introduced to describe the features of this PRG flow:

$E \bullet J=\frac{v}{g}\left(1+\frac{v \beta d}{n v}\right) v$

where $E$ is permeability coefficient tensor $\left(\mathrm{m}^{2}\right) ; J$ is the fluid pressure gradient $(\mathrm{Pa} / \mathrm{m}) ; v$ is the cohesion coefficient $\left(\mathrm{m}^{2} / \mathrm{s}\right)$; $\mathrm{G}$ is the gravity acceleration $\left(9.8 \mathrm{~m} / \mathrm{s}^{2}\right) ; v$ is the fluid velocity $(\mathrm{m} / \mathrm{s}) ; \beta$ is the form factor of medium particles; $\mathrm{d}$ is the average particle size $(\mathrm{m}) ; n$ is porosity of the porous medium $(\%)$.

\subsection{State equation of the PRG flow}

During the mining, the PRG flow is mainly caused by the pressure gradient. Here, the PRG is regarded as an ideal gas, and the temperature is considered as isothermal. Then, the state equation of the PRG flow can be expressed as:

$\frac{\rho}{\rho_{0}}=\frac{p}{p_{0}}$

where $\rho$ and $\rho_{0}$ are the gas densities at the pressures of $\rho$ and $p_{0}$, respectively. The equation shows that the gas density varies dynamically with gas pressure.

\subsection{Content equation of coal seam gas}

In mining conditions, a lot of adsorbed gas will be desorbed to generate free gas. Then, the free gas flowing through the micro pores and fractures in the coal seam will vary with the gas pressure and temperature. Hence, the amount of free gas stored in the coal seam can be expressed as:

$M_{y}=\frac{p z_{0} T_{0}}{z T} \phi$

The adsorbed gas in the coal seam can be calculated by the Langmuir adsorption equation:

$M_{x}=\frac{a b p}{1+b p} c$

Therefore, total content of gas in the coal seam can be derived as:

$M=M_{x}+M_{y}=\frac{a b p}{1+b p} c+\frac{p z_{0} T_{0}}{z T} \phi$

\section{NUMERICAL SIMULATION OF PRG FLOW}

\subsection{Establishment of physical model}

Based on some reasonable assumptions, the Ansys Fluent software was employed to model the coalface, goaf, overlying coal-rock and underlying coal-rock in Longfeng Coalmine in northeastern China's Liaoning Province. The modelling effect is shown in Figure 1.

In the model, there are a total of 13 coal seams, denoted as $1 \# \sim 13 \#$. In the model, there are a total of 13 coal seams, 
denoted as $1 \# \sim 13 \#$. Among them, coal seams 1\# 9\# belong to the overlying coal-rock ( $43 \mathrm{~m}$ in height), while coal seams $10 \# \sim 13 \#$ belong to the underlying coal-rock ( $33 \mathrm{~m}$ in height).

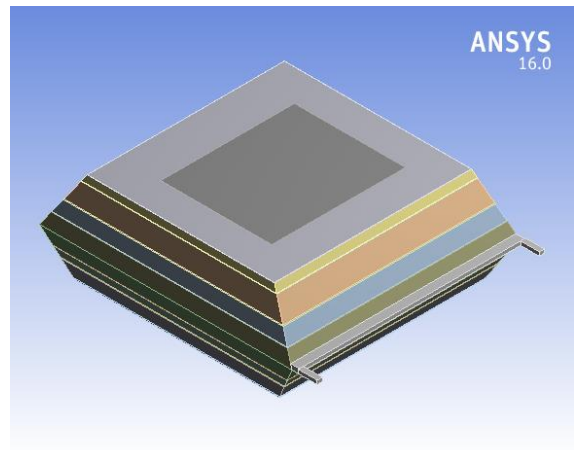

Figure 1. Physical model of the coalface

\subsection{Gas flow during buried pipe extraction in the goaf}

Figure 2 shows the simulated results of buried pipe extraction, and Figure 3 shows the iso-concentration map of the gas at the floor of the goaf.

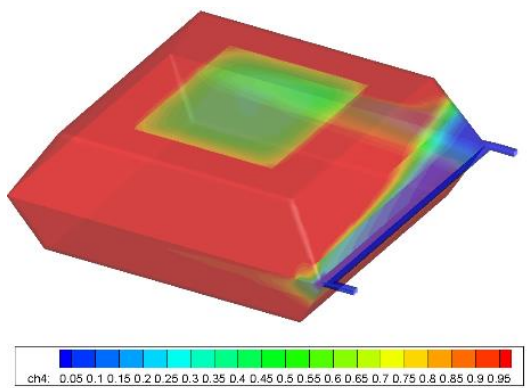

Figure 2. Spatial distribution of gas concentration

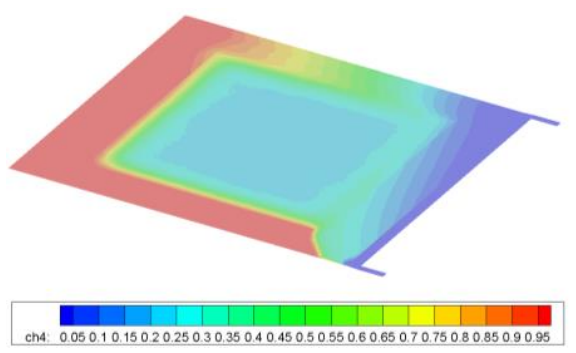

Figure 3. Iso-concentration map of the gas at the floor of the goaf

It can be seen from Figure 3 that the gas concentration in the goaf gradually decreases from the deeper layer to the coalface after the mining of coal seam 9\#. Meanwhile, the air leakage in the intake airway, together with the gravitational acceleration, has an impact on the return airway: a large amount of dense gas accumulates in the strip on the side of the return airway. Within $30 \mathrm{~m}$ from the return airway, the gas concentration gradient changes significantly, with the concentration ranging from $10 \%$ to $95 \%$. In this area, the gas is less dense in locations farther away from the coalface on the side of the return airway.

In the deep goaf over $30 \mathrm{~m}$ away from the workface on the side of the return airway, the gas concentration $(>70 \%)$ is only slightly influenced by the air leakage in the intake airway. Due to the negative pressure of the buried pipe, the gas in this area has a deeper concentration gradient near the return airway of the goaf, with the concentration ranging from $0 \%$ to $10 \%$ near the upper corner of the coalface. The high gas content in that corner restricts normal mining, adding to the difficulty in gas treatment.

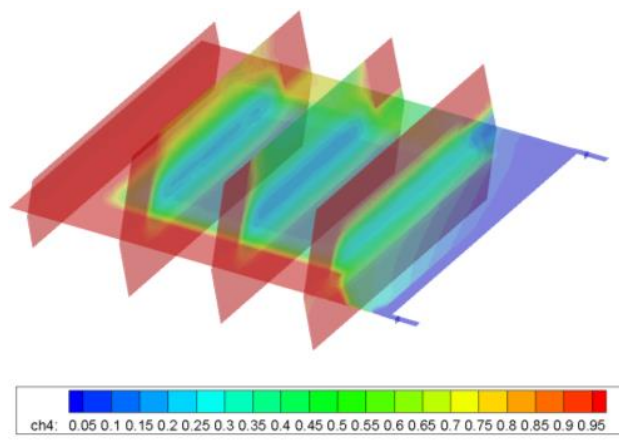

Figure 4. Space slices in the mining direction

As shown in Figure 4, along the mining direction, the gas concentration in the overlying coal-rock is positively correlated with the distance from the coalface. As for the underlying coal-rock, the PRG in that mass flows upward to the goaf under the buoyancy force, and accumulates near the return airway along the air flow direction in the goaf. Hence, the return airway has a high amount of gas, making it hard to drain the gas.

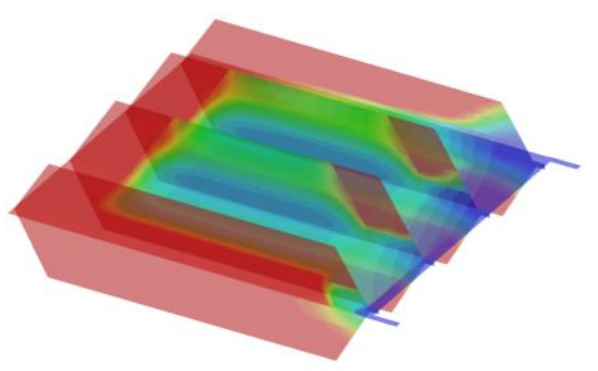

che: 0.050 .10 .150 .20 .250 .30 .350 .40 .450 .50 .550 .60 .650 .70 .750 .80 .850 .90 .95

Figure 5. Space slices on gas concentration

As shown in Figure 5, the gas concentration in the overlying coal-rock tends to increase from the intake airway to the return airway. The reason for this trend is explained as follows. In places close to the coalface, there is a great amount of leaked air such that the RPG flows along the air flow direction; in places far away from the coalface, the air flows rather slowly because of the small leakage amount, resulting in a limited gradient of gas concentration.

Similarly, the gas concentration in the underlying coal-rock also gradually increases from the intake airway to the return airway. The goaf is about $30 \sim 50 \mathrm{~m}$ away from the return airway. The gas concentration increases at about $20 \mathrm{~m}$ above the nearby coal seam 5\%, making it more likely for gas to accumulate on the upper corner. Comparatively, coal seam 13\#, the floor of the underlying coal-rock, has the highest gas concentration.

From the coal-rock close to the coalface, the PRG moves upward through the following steps: the gas flows into the goaf, moves along with the leaked air, returns to the side of the return airway, and accumulates in the upper corner. The distribution of gas concentration shows that the return airway is thronged with gas, especially near the upper corner.

To prevent gas overrun and leakage, the gas concentration at this position is normally controlled by buried pipe extraction. 
However, a single round of extraction may not be enough to deal with severe gas leakage. If the amount of gas is excessively high in the upper corner, it is necessary to carry out several rounds of buried pipe drainage to achieve a desirable treatment effect.

\section{CONCLUSION}

Taking the coalface of Longfeng Coalmine as the object of Fluent numerical simulation, the author set up a gas movement model for the coalface, and analysed the simulated results. The following conclusions were drawn from the research:

(1) The gas concentration in the overlying coal-rock tends to increase from the intake airway to the return airway. Within $30 \mathrm{~m}$ from the return airway, the gas concentration gradient changes significantly, with the concentration ranging from $10 \%$ to $95 \%$.

(2) Similarly, the gas concentration in the underlying coalrock also gradually increases from the intake airway to the return airway. The PRG moves upward through the following steps: the gas flows into the goaf, moves along with the leaked air, returns to the side of the return airway, and accumulates in the upper corner.

(3) Under the joint action of air leakage, gravitational acceleration and buoyancy, the PRG flows from the overlying and underlying coal-rock masses, and accumulates near the top coal seam $1 \#$, the coal seam $5 \#$ at the side of the return airway, and the bottom coal seam $13 \#$.

\section{ACKNOWLEDGMENT}

I would like to express my gratitude to all those who helped me during writing of this thesis. And thank you to all the authors of references, thank you for your inspiration and guidance in my essay writing.

\section{REFERENCES}

[1] Hu Q.T., Zhao X.S. (2012). Status of coal and gas outburst accidents in China and suggestions for prevention, Mining Safety and Environmental Protection, Vol. 5, pp. 1-6, 99. DOI: 10.3969/j.issn.1008-4495.2012.05.001

[2] Liu W.G., Hu Y.H. (2003). Present status and potential of mine ventilation gas utilization technology, China Coal, Vol. 11, pp. 11-12. DOI: 10.3969/j.issn.1006530X.2003.11.003

[3] Chen Y., Yang C.M. (2008). The inspire of overseas coal-bed methane's exploitation and utilization, China Mining Magazine, Vol. 17, No. 4, pp. 11-14. DOI: 10.3969/j.issn.1004-4051.2008.04.004

[4] Zupanick J.A. (2006). Coal mine methane drainage using multilateral horizontal wells, Mining
Engineering, Vol. 58, No. 1, pp. 50-52.

[5] Szlązak N., Swolkień J. (2016) The effectiveness of the methane drainage of rock-mass with a $\mathrm{u}$ ventilation system, Archives of Mining Sciences, Vol. 61, pp. 617634. DOI: $10.1515 / \mathrm{amsc}-2016-0044$

[6] Holla L., Buizen M. (1991). The ground movement, strata fracturing and changes in permeability due to deep longwall mining, International Journal of Rock Mechanics and Mining Sciences, Vol. 28, pp. 207-217. DOI: 10.1016/0148-9062(91)92168-X

[7] Bogert H., Jung S.J., Lim H.W. (1997). Room and pillar stope design in highly fractured area, International Journal of Rock Mechanics and Mining Sciences, Vol. 34, No. 3, pp. 145-156. DOI: 10.1016/S1365-1609(97)00155-X

[8] Mc Tigue D.F., Mei C.C. (1987). Gravity induced stresses near axisymmetric topography of small slopes, Int J Num Anal Meth Geomech, Vol. 11, pp. 257-268. DOI: $\frac{10.1002 / \text { nag.1610110304 }}{\text { X.X. }}$

[9] Miao X.X., Qian M.G. (2009). Research on green mining of coal resources in China: Current status and future prospects, Journal of Mining and Safety Engineering, Vol. 1, pp. 1-14. DOI: 10.3969/j.issn.1673-3363.2009.01.001

[10] Zhai C. (2008). Research on the coupling laws between fissure field and gas flow field of short-distance coal seams group and preventive technology, China University of Mining and Technology.

[11] Wang D.S. (2009). Theories and test of stereo gas drainage for outburst prevention under closed distance seam group with protection layer mining, China University of Mining and Technology.

[12] Liu H.Q. (2001). Survey study on the influence laws of the floor over pressure aquifer by harmonic mining, Journal of Xuzhou Institute of Architectural Technology, Vol. 2, pp. 12-15. DOI: 10.3969/j.issn.2095-3550.2001.02.004

[13] Miao X.X., Liu W.Q., Chen Z.Q. (2004). Mining Rock Mass Seepage Theory, Science Press, Beijing.

[14] Gray I. (1987). Reservoir engineering in coal seams, SPE Reservoir Engineering, pp. 28-34

[15] Li X.C., Kang Y.L., Chen D.F., Chen F. (2014). Effect of drill-in fluids on CBM desorption, diffusion and percolation: A case study on NO. 9 coal seam of the Ningwu basin, Natural Gas Industry, Vol. 34, No. 1, pp. 86-91. DOI: $10.3787 /$ j.issn.1000-0976.2014.01.013

[16] Sun K.M. (2005). Fluid-solid coupling theory of exploiting coal methane and improving production by gai injection in low permeability reservoir and its application, Chinese Journal of Rock Mechanics and Engineering, Vol. 12, pp. 2081. DOI: 10.3321/j.issn:1000-6915.2005.12.033

[17] Wei J.P., Qin H.J., Wang D.K., Yao B.H. (2015). Dynamic permeability model for coal containing gas, Journal of China Coal Society, Vol. 40, No. 7, pp. 1555-1561. DOI: $10.13225 /$ j.cnki.jccs.2014.1376 